\title{
Risk of tuberculosis in low birth weight children from East Romania
}

\author{
Elena Ariela Banu ${ }^{1}$, Aurel Nechita ${ }^{1}$, Eva Maria Elkan-Cojocaru ${ }^{1}$, Ginel Baciu ${ }^{1}$, Alina Manole², \\ Liliana Chelaru ${ }^{3}$
}

\author{
${ }^{1}$ Faculty of Medicine and Pharmacy, "Dunărea de Jos" University, Galați; "Sf. Ioan” \\ Emergency Clinic Hospital for Children, Galați, Romania \\ 2Department of Preventive Medicine and Interdisciplinarity, "Grigore T. Popa" \\ University of Medicine and Pharmacy, Iași, Romania \\ ${ }^{3}$ Department of Morphological Sciences, “Grigore T. Popa” University of Medicine \\ and Pharmacy, Iași, Romania
}

Submitted: 22 June 2018

Accepted: 30 August 2018

Arch Med Sci 2020; 16 (1): 162-166

DOI: https://doi.org/10.5114/aoms.2018.78768

Copyright (c) 2018 Termedia \& Banach

\author{
Corresponding author: \\ Alina Manole MD, PhD \\ Department of \\ Preventive Medicine \\ and Interdisciplinarity \\ "Grigore T. Popa" \\ University of Medicine \\ and Pharmacy \\ 16 Universitatii St \\ 700115 Iași, Romania \\ Phone: +40748656676 \\ E-mail: alina.manole@ \\ umfiasi.ro
}

\begin{abstract}
Introduction: In the context of the global tuberculosis (TB) burden, children represent $10 \%$ of all cases, with high incidence rates still reported by many regions worldwide. The study aim was to determine whether there is a correlation between TB clinical diagnosis and low birth weight in children at various ages.

Material and methods: The study was conducted between 2010 and 2014, on a group of 1783 pediatric patients and a subgroup of 137 pediatric patients with low birth weight (LBW). Data were collected from patients' records and hospital statistical reports then processed using MS Excel 2010 and SPSS v.22.

Results: The subgroup of LBW patients accounted for $7.68 \%$ of all recorded cases. Girls were predominant (total $M: F=0.95$; LBW group $M: F=0.91$, $p<0.05$ ), most from an urban area (total $\mathrm{U}: R=1.29$; LBW subgroup $\mathrm{U}$ : $R=1.36, p<0.05) .22 .59 \%$ of LBW subgroup children were infants aged of 0-12 months. The youngest age at TB diagnosis was 1 month and the lowest weight was $700 \mathrm{~g}$. ANOVA regression for LBW and age at TB diagnosis, showed a multiple $R$ value of $0.0256, p=0.7659(F=0.7659,95 \% \mathrm{Cl})$.

Conclusions: The correlation between clinical diagnosis of tuberculosis in children at various ages and their low birth weight was positive but was not statistically significant. However, this research hypothesis should be tested in further studies on larger population groups, due to the current public health context of "End TB", promoted worldwide.
\end{abstract}

Key words: tuberculosis, epidemiology, pediatrics, low birth weight.

\section{Introduction}

\section{The epidemiological context and public health impact of tuberculosis}

According to the World Health Report, the number of new cases of TB was approximately 10.4 million, of which $10 \%$ were in children. Eastern Europe recorded fewer cases (7\%) and other regions of Europe recorded $3 \%[1,2]$. The WHO Global Strategy of "End TB" and the United Nations Sustainable Development Goals for the period 2016-2035 share the end- 
ing of the global TB epidemic [3-5]. At the global level, children aged under 15 years accounted for $6.9 \%$ of new cases of TB noted in 2016 (approximately 1 million cases). In children, regardless of age, TB is currently the leading cause of infectious death. Children with compromised immune status, such as infants, preterm newborns, HIV-infected or seriously malnourished, are most at risk of TB. Risk factors often include poor populations without access to healthcare facilities. Although $\mathrm{TB}$ is curable, the diagnostic methods are imperfect and have poor performance in children due to the difficulty of obtaining evidence and low bacillary loads. Adolescents have a particular risk of developing an adult-like clinical form; that is, sputum is often positive and highly infectious [1, 6, 7].

For Romania, the year 2016 reports showed that, for a population of 20 million people, the overall global incidence of TB was 15,000 cases, with a mean of $74 \% 000$, and MDR-TB cases numbered approximately 4500 (3.8\%000). New and relapsed TB cases as notification rates by age group, during 2007-2016, showed a constant trend [8].

\section{Research hypothesis}

Previous studies in animals have shown that the fetus can adapt to an adverse intrauterine environment by slowing down growth and metabolism. This adaptive strategy seems to have long-term adverse health consequences. Researchers proved that genetic factors could influence birth size but also diseases that occur later in life. Epigenetic factors (environment, smoking, alcohol consumption, and other health conditions of ascendants) are thought to influence the occurrence of low birth weight as well as the specificity of child and adult pathology along with classical genetic and non-genetic factors currently known [9].

A study conducted in Sweden on a large group of twins revealed an association between birth weight or weight and TB. The study authors highlighted in conclusion that fetal growth might have an association with susceptibility to TB due to the hypothesis of early programming immunity [10].

The study aim was to determine whether there is a correlation between TB clinical diagnosis and low birth weight in children at various ages, taking into account the epidemiological context of TB in the last decade.

\section{Material and methods}

The study was conducted between 2010 and 2014, on a group of 1783 patients admitted to the Hospital of Pneumophthisiology (Pediatric Pneumology Unit) and "Sf. Ioan" Clinical Emergency Hospital for Children, in Galați County, Romania.
The inclusion criteria were pediatric age, the clinical diagnosis of TB and the birth weight noted in patients' records. The total number of admitted cases was 1873 from a previous study [11], but, because of certain omitted data, 66 records had to be eliminated. The definition of low birth weight (LBW) was considered to be less than $2500 \mathrm{~g}$ [12]. According to this limit, we selected another subgroup of 137 pediatric patients with birth weights $<2500 \mathrm{~g}$. The database of patients' records did not specify whether LBW was in a small for gestational age or preterm newborn. The exclusion criteria did not involve residence area, gender or other comorbidities, so the variables established for the study were the following: age, gender, residence area, and birth weight. All the pediatric patients as well as their mothers had HIV-negative status during pregnancies [13].

Data were obtained in accordance with a request letter approved and signed by the medical manager committee of the Hospital of Pneumophthisiology and Galati Public Health Authority. Data were collected from patients' records and hospital statistical reports then processed using MS Excel 2010 and SPSS v.22.

\section{Results}

\section{Descriptive comparison between the two groups}

The distribution of cases by years of study showed statistically insignificant variation between years, with a minimum of 317 cases in 2013 and a maximum of 395 cases in 2014, mean = 356.6. For the subgroup of patients with LBW, they accounted for $7.68 \%$ of all cases, with a minimum of 20 cases in 2012 and a maximum of 32 in 2013, mean $=27.4$ (Figure 1 ).

Distribution by gender showed that girls were predominant, with a total $M: F=0.95$ and LBW group $M: F=0.91$ but with no statistically significant male/female differences in almost all years of the study $(p>0.05)$ (Figure 2).

According to the residence area, there was a statistically significant difference $(p<0.05)$ between urban and rural patients both in the total

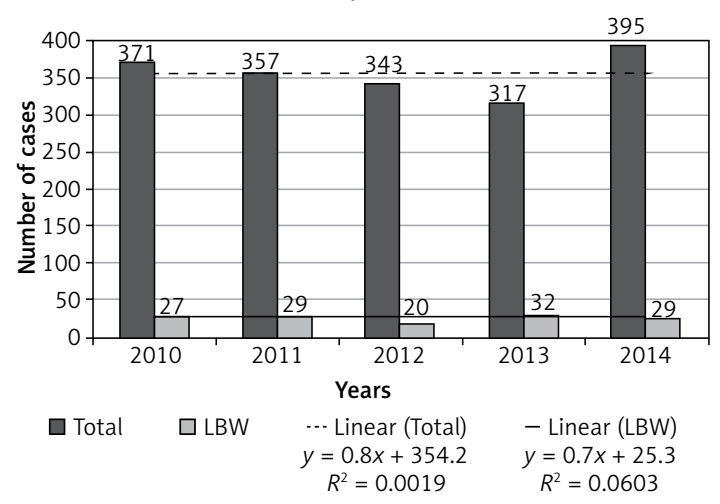

Figure 1. Distribution of cases by years of study 


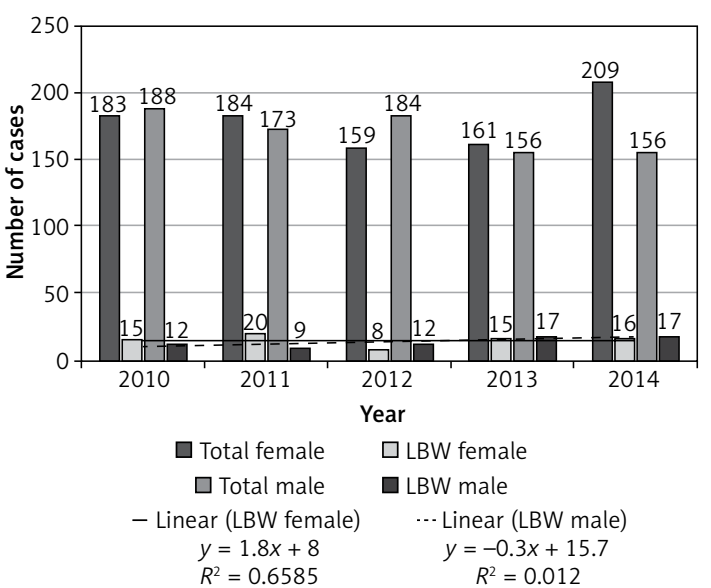

Figure 2. Distribution of cases by gender

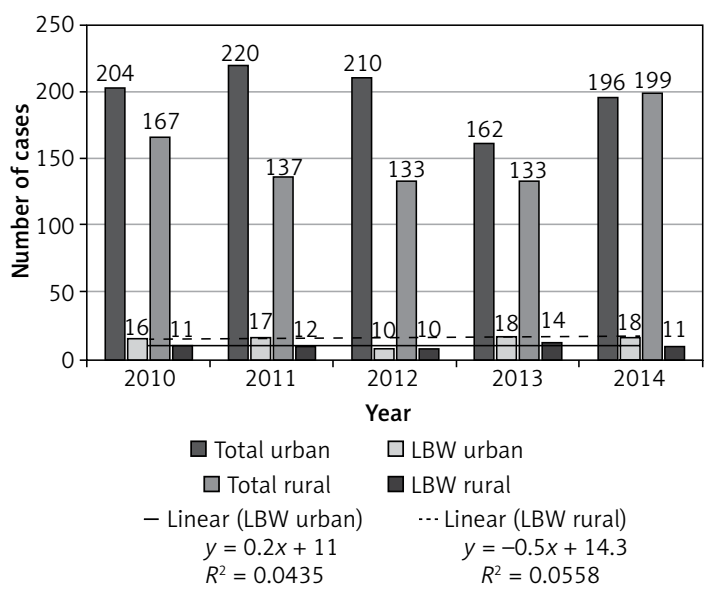

Figure 3. Distribution of cases by residence area

Table I. Distribution of cases by age group

\begin{tabular}{|lcccccccccc|}
\hline \multirow{2}{*}{$\begin{array}{l}\text { Year of } \\
\text { study }\end{array}$} & \multicolumn{10}{c|}{ Distribution by age group } \\
\cline { 2 - 14 } & \multicolumn{2}{c}{$\mathbf{0 - 1}$ year } & \multicolumn{2}{c|}{ 2-4 years } & \multicolumn{2}{c|}{ 5-9 years } & 10-14 years & \multicolumn{1}{c|}{ 15-18 years } \\
\cline { 2 - 14 } & Total & LBW & Total & LBW & Total & LBW & Total & LBW & Total & LBW \\
\hline 2010 & 14 & 6 & 81 & 9 & 141 & 5 & 69 & 5 & 66 & 2 \\
\hline 2011 & 9 & 2 & 99 & 6 & 105 & 7 & 74 & 6 & 70 & 8 \\
\hline 2012 & 17 & 3 & 80 & 4 & 97 & 4 & 86 & 6 & 63 & 3 \\
\hline 2013 & 9 & 2 & 60 & 5 & 101 & 9 & 85 & 9 & 62 & 7 \\
\hline 2014 & 13 & 1 & 59 & 6 & 114 & 6 & 112 & 11 & 96 & 5 \\
\hline Total & 62 & 14 & 379 & 30 & 558 & 31 & 426 & 37 & 357 & 25 \\
\hline
\end{tabular}

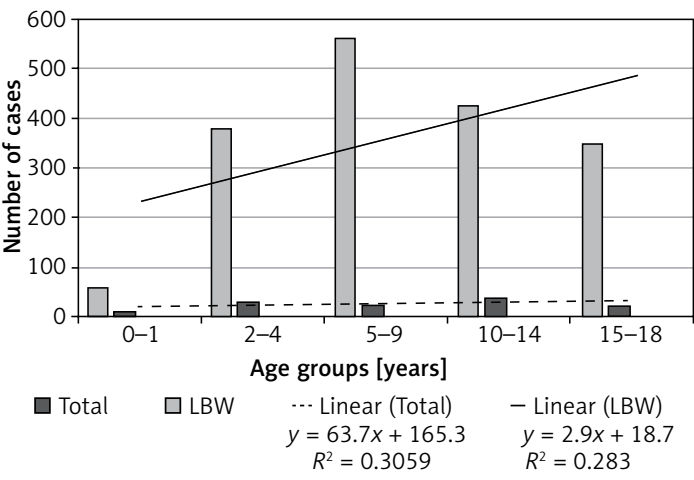

Figure 4. Distribution of total cases by age groups

group, with a total $U: R=1.29$, and in the LBW group, with a $U: R=1.36$ (Figure 3 ).

Distribution by pediatric age groups showed a proportion of $22.59 \%$ of $0-1$ year old children with low birth weight diagnosed with TB, while in other age groups, the proportions were much more decreased, such as: $7.91 \%$ of 2-4 year old children; $5.55 \%$ of the $5-9$ age group; $8.68 \%$ in children aged $10-14$; and $7.01 \%$ of $15-18$ aged patients (Table I, Figure 4).

\section{Low birth weight group statistics}

Descriptive statistical data regarding age and weight for the LBW group evidenced that the youngest age at TB diagnosis was 1 month and the lowest weight was $700 \mathrm{~g}$, with the mean age of 8.59 and the median age of 8 . After applying ANOVA regression for LBW and age at TB diagnosis, the following results were obtained: multiple $R=0.0256$, $p=0.7659(F=0.7659,95 \% \mathrm{Cl})$. Although tests revealed no statistical significance, a positive correlation was found between low birth weight and the risk of TB occurrence in pediatric ages.

\section{Discussion}

The results of our study did not provide significant statistical evidence to assert that low birth weight represents a risk for further TB infection.

However, a cohort study performed in Sweden examined the risk of TB in relation to birth weight in a group of 21,596 twins born between 1926 and 1958. The authors found that the risk of TB was $11 \%$ lower for every $500 \mathrm{~g}$ of birth weight, with a stronger correlation found in male subjects. Because associations among monozygotic twins are largely independent of genetic or environmental factors, the authors claimed that fetal growth may play a causal role in susceptibility to TB, possibly due to early immunity system programming [10]. 
It is also known that LBW is associated with a greater number of hospitalizations in infectious disease hospitals during childhood. These children with a lower immunity are at risk of coming into closer contact with sources and contaminated transmission routes, in a variety of possible carriers. Some studies have even reported a correlation between normal birth weight and the risk of certain conditions over the lifetime. The specialists in the field stated that, however, there is currently no consensus on the limits of normal weight at birth, using the official lower limit of 2,500 g. There were researchers who argued that the optimal birth weight for a healthy future child and adult would be 3500-4500 g. Little is known about what the risk is for a child who is neither LBW nor optimal weight [14]. We consider that such a study on an in-between birth weight group risk associated with TB will be a further investigation of our research team.

Recent estimates of the incidence of pediatric TB suggest that figures are much higher than previously estimated or are directly reflected in the notification dates, with more than $60 \%$ being undeclared or undiagnosed cases. The magnitude of sub-diagnostics in children and the restrictions on the availability and quality of direct data at the population level lead to a substantial uncertainty that is difficult to quantify in estimates based on such data sources [15-17].

TB remains a diagnostic problem, especially in children with clinical lymph nodes and lack of evidence for Mycobacterium tuberculosis, especially for differential diagnosis of head and neck formations [18]. Studies have shown that susceptibility to $M$. tuberculosis infection is influenced by many factors such as malnutrition (including low birth weight), HIV infection and genetic factors such as interleukin and vitamin $D$ receptor, but also environmental factors such as the social and financial level of the individuals and the community [19].

Clinical symptoms of TB in neonates are nonspecific and their parents are often asymptomatic, leading to a delayed diagnosis and a long period when the child is contagious and without specific treatment to prevent secondary transmission. Such a case in a neonatology intensive care unit may transmit the disease not only to newborns or to medical staff, but also to those in the family or other individuals who come into contact with the newborn. In Japan, such a case of TB was reported in a very low birth weight newborn from a pregnant woman who had had TB-like symptoms at 8 weeks of pregnancy [20].

Authors from Germany reported a possible case of congenital TB in a premature infant with an extremely low birth weight of $460 \mathrm{~g}$, with a mother who was diagnosed with TB endometritis immediately after delivery. Mycobacterium tuberculosis was detected from the infant's gastric juice, at 156 days of age. The authors asserted that there was no evidence for horizontal transmission. Both mother and child were treated favorably. This case report highlighted a prolonged latency period of TB infection, emphasizing the need for urgent treatment of neonates in such situations [21], especially taking into account pediatric acute respiratory failure [22].

Although for over two decades efforts have been made to prevent and treat TB, there are still over 10 million new cases reported and nearly 2 million deaths annually. If one-fourth of the global population appears to be infected with $M$. tuberculosis, the Universal Health Coverage concept also depends on optimizing the methods of prevention, screening, treatment, epidemiological surveillance, and the real knowledge of risk factors in order to reduce the incidence of TB over $17 \%$ by $2025[23,24]$.

The Bacillus Calmette-Guérin (BCG) vaccine, used for almost a century, is still widely in use and prevents the emergence of severe TB in children [1].

Children with LBW are thought to have a lower immune response than others, and this may influence the effectiveness of their subsequent BCG vaccination. Recovering infants with $L B W$ on $B C G$ vaccination could be a problem in our country, especially in rural areas. Pediatric patients included in our study were not vaccinated during the first year of life or the effectiveness of BCG vaccine was inconclusive [14].

The reported figures and their insignificant decreases from 1 year to another, as well as the pediatric TB burden with its epidemiological and clinical consequences, confirm once again the statement made half a century ago about the important contributions a pediatrician might have to the fight against TB, considering that wherever there are adult TB patients, there are infected children, too. No child will be absolutely protected against TB until the incidence of TB is reduced to the point where it is not a public health problem anymore [15].

The limitation of our study is first of all the quite small number of patients, in order to obtain statistically significant results. Another limitation is represented by incomplete hospital recordings. Because there was no interview with patients or their family members, our variables included in the database were limited to basic data which are mentioned currently in an admission record.

In conclusion, the correlation between clinical diagnosis of TB in children at various ages and their low birth weight was positive but not statistically significant. However, this research hypothesis could be tested in further studies on larger population groups, due to the current public health context of "End TB", promoted worldwide. 


\section{Acknowledgments}

The authors wish to thank the medical staff of the Hospital of Pneumophthisiology, Pediatric Pneumology Unit, for their help in collecting data. We declare no financial relationship with any organizations or industry.

\section{Conflict of interest}

The authors declare no conflict of interest.

\section{References}

1. World Health Organization. Global tuberculosis report 2017. Geneva: World Health Organization; 2017. Licence: CC BY-NCSA 3.0 IGO

2. Erdem B, Peker N, Inci A, et al. Pelvic tuberculosis mimicking ovarian cancer: a series of 13 cases. Arch Med Sci Civil Dis 2017; 2: 177-81.

3. Uplekar M, Weil D, Lonnroth K, et al. WHO's new End TB Strategy. Lancet 2015; 385: 1799-801.

4. World Health Organization. Implementing the End TB Strategy: the essentials. Geneva: World Health Organization, 2016.

5. Hogan DR, Stevens GA, Hosseinpoor AR, Boerma T. Monitoring universal health coverage within the Sustainable Development Goals: development and baseline data for an index of essential health services. Lancet Glob Health 2018; 6: e152-68.

6. Dodd PJ, Yuen CM, Sismanidis C, Seddon JA, Jenkins HE. The global burden of tuberculosis mortality in children: a mathematical modelling study. Lancet Global Health 2017; 5: e898-906.

7. UNICEF 2017. Is Every Child Counted? Status of data for children in the SDGs. New York. 2017.

8. European Centre for Disease Prevention and Control/ WHO Regional Office for Europe. Tuberculosis surveillance and monitoring in Europe 2018-2016 data. Stockholm: ECDC; 2018.

9. Risnes KR, Vatten LJ, Baker JL, et al. Birthweight and mortality in adulthood: a systematic review and metaanalysis. Int J Epidemiol 2011; 40: 647-61.

10. Villamor E, lliadou A, Cnattingius S. Evidence for an effect of fetal growth on the risk of tuberculosis. J Infect Dis 2010; 201: 409-13.

11. Banu EA. Scorpan C, Mihailov O, et al. Epidemiological aspects of pediatric tuberculosis in Eastern part of Romania. Rev Med Chir Soc Nat lasi 2017; 121: 763-9.

12. World Health Organization. Newborns with low birth weight. Available at: http://www.who.int/whosis/whostat 2006NewbornsLowBirthWeight.pdf. Accessed on 31.03.2018.

13. Lin HC, Lin HC, Chen SF. Increased risk of low birthweight and small for gestational age infants among women with tuberculosis. BJOG 2010; 117: 585-90.

14. Bellingham-Young DA, Adamson-Macedo EN. The impact of birthweight on adult minor illness: a study on a sub-clinical population. J Human Growth Develop 2013; 23: 11-7.

15. Graham SM, Sismanidis C, Menzies HJ, Marais BJ, Detjen AK, Black RE. Importance of tuberculosis control to address child survival. Lancet 2014; 383: 1605-7.

16. Oliwa JN, Karumbi JM, Marais BJ, Madhi SA, Graham SM. Tuberculosis as a cause or comorbidity of childhood pneumonia in tuberculosis-endemic areas: a systematic review. Lancet Respir Med 2015; 3: 235-43.
17. World Health Organization. Roadmap for childhood tuberculosis: towards zero deaths. Geneva: World Health Organization; 2013.

18. Bruzgielewicz A, Rzepakowska A, Osuch-Wójcikewicz E, Niemczyk K, Chmielewski R. Tuberculosis of the head and neck - epidemiological and clinical presentation. Arch Med Sci 2013; 10: 1160-6.

19. Cao Y, Wang X, Cao Z, Cheng X. Vitamin D receptor gene Fokl polymorphisms and tuberculosis susceptibility: a meta-analysis. Arch Med Sci 2016; 12: 1118-34.

20. Saitoh M, Ichiba H, Fujioka H, Shintaku H, Yamano T. Connatal tuberculosis in an extremely low birth weight infant: case report and management of exposure to tuberculosis in a neonatal intensive care unit. Eur J Pediatr 2001; 160: 88-90.

21. Vogel M, Schroten H, Kahl P, Müller A. High latency of tuberculosis manifestation in a premature extremely low birth weight infant with favorable outcome. Neonatology 2014; 105: 91-4.

22. Elsamahy M, Abdelmonem M, Ibrahim H, Eldin M, Allam A. Success or failure of non-invasive positive pressure ventilation in children with acute respiratory failure. Could it be predicted? Arch Med Sci Civil Dis 2017; 2: 113-20.

23. Leung CC, Chee CBE, Zhang Y. Tuberculosis updates 2018: innovations and developments to end TB. Respirology 2018; 23: 356-8.

24. Houben RM, Dodd PJ. The global burden of latent tuberculosis infection: a re-estimation using mathematical modelling. PLoS Med 2016; 13: e1002152. 